\title{
Interference enhanced thermoelectricity in quinoid type structures
}

\author{
M. Strange, ${ }^{1, a)}$ J. S. Seldenthuis, ${ }^{2}$ C. J. O. Verzijl, ${ }^{2}$ J. M. Thijssen, ${ }^{2}$ and G. C. Solomon ${ }^{1}$ \\ ${ }^{1}$ Nano-Science Center and Department of Chemistry, University of Copenhagen, Universitetsparken 5, \\ 2100 Copenhagen $\emptyset$, Denmark \\ ${ }^{2}$ Kavli Institute of Nanoscience, Delft University of Technology, 2628 CJ Delft, The Netherlands
}

(Received 19 November 2014; accepted 10 February 2015; published online 25 February 2015)

\begin{abstract}
Quantum interference (QI) effects in molecular junctions may be used to obtain large thermoelectric responses. We study the electrical conductance $G$ and the thermoelectric response of a series of molecules featuring a quinoid core using density functional theory, as well as a semi-empirical interacting model Hamiltonian describing the $\pi$-system of the molecule which we treat in the GW approximation. Molecules with a quinoid type structure are shown to have two distinct destructive QI features close to the frontier orbital energies. These manifest themselves as two dips in the transmission, that remain separated, even when either electron donating or withdrawing side groups are added. We find that the position of the dips in the transmission and the frontier molecular levels can be chemically controlled by varying the electron donating or withdrawing character of the side groups as well as the conjugation length inside the molecule. This feature results in a very high thermoelectric power factor $S^{2} G$ and figure of merit $Z T$, where $S$ is the Seebeck coefficient, making quinoid type molecules potential candidates for efficient thermoelectric devices. (ㄷ 2015 AIP Publishing LLC. [http://dx.doi.org/10.1063/1.4913290]
\end{abstract}

\section{INTRODUCTION}

Calculations suggest that quantum interference (QI) effects in molecules give rise to a large tunability of the electron transport properties of molecular junctions. These effects induce a strong variation of the transmission with energy, which is favourable for thermoelectricity, where an electric current or potential difference develops in response to a temperature difference across the molecule. ${ }^{1-3}$ Well studied QI molecular units usually involve either cross conjugation and/or meta-coupled phenyl units. ${ }^{4-8}$ Molecules such as anthraquinone have recently been shown to exhibit destructive QI (DQI) effects in a molecular junction. ${ }^{9,10}$ A simple nearestneighbour tight-binding model of the $\pi$ system has been used to rationalise the QI effects. Moreover, schemes have been developed to make predictions based on simple graphical rules. ${ }^{11,12}$ The sign of the thermopower has been suggested to provide information of whether the transport is mainly via occupied- or unoccupied molecular states. ${ }^{1}$ To obtain a strong thermoelectric response, the destructive QI feature needs to be close to the Fermi level. A sensible handle to control the position of QI features is offered by tuning the chemistry of the binding groups or changing the electronegativity of substituent side groups, which has been shown theoretically as well as experimentally to influence the thermopower by changing the molecular frontier levels relative to the Fermi level of the metal electrodes. ${ }^{13-17}$

Cruciform oligo(phenylene ethynylene)s (OPEs) with a conjugated and extended tetrathiafulvalene (TTF) donor moiety have recently been synthesised ${ }^{18,19}$ and shown to be redox active as well as having interesting spin properties in the Coulomb blockade regime. ${ }^{20}$ Cruciform type molecules have

\footnotetext{
a)Electronic mail: strange@ @chem.ku.dk
}

also been synthesised with substituent side groups, such as TTF, dithiofulvalene (DTF), and atomic oxygen, forming crossconjugated OPEs. Such structures can be referred to as quinoid, since the central core corresponds to a quinone, with the substituents replacing the oxygen atoms, see Figures 1(a) and 5. They may exist as zwitterions with mixed substituents, an electron donor on one side and an electron accepting group on the other side. This suggests the possibility of electric field induced switching between the conjugated (high conductance) and cross-conjugated (low conductance) state. While previous theoretical studies have found that the thermoelectric response of molecules may be greatly enhanced by QI effects, they involved either rather long molecular wires, radicals, or metal complexes without anchoring groups. ${ }^{2,3,21}$

Here, we explore the electron transport properties of cruciform molecules with a quinoid type structure by varying the electron donating (ED) and electron withdrawing (EW) character of substituent side groups. We study the low bias conductance $G$ as well as the ability of the molecules to convert thermal energy into electric energy by applying a temperature difference across the molecular junction. We find, based on density functional theory (DFT) calculations, that quinoid structures show two characteristic DQI features, one near the highest occupied molecular orbital (HOMO) level and one near the lowest unoccupied molecular orbital (LUMO) level. The DQI features result not only in a high thermopower (Seebeck coefficient) $S$, but more importantly from the point of view of technological application, a high power factor (PF), $S^{2} G$. The PF is related to the electrical work that can be extracted from a thermoelectric device and is the quantity that determines the dimensionless figure of merit, $Z T=S^{2} G T /\left(\kappa_{\mathrm{ph}}\right.$ $\left.+\kappa_{\mathrm{el}}\right)$, when the phonon thermal conductance $\kappa_{\mathrm{ph}}$ dominates over the electron thermal conductance $\kappa_{\mathrm{el}}{ }^{22-24}$ We find that a semi-empirical model for the $\pi$ system, treated in the GW 
(a)
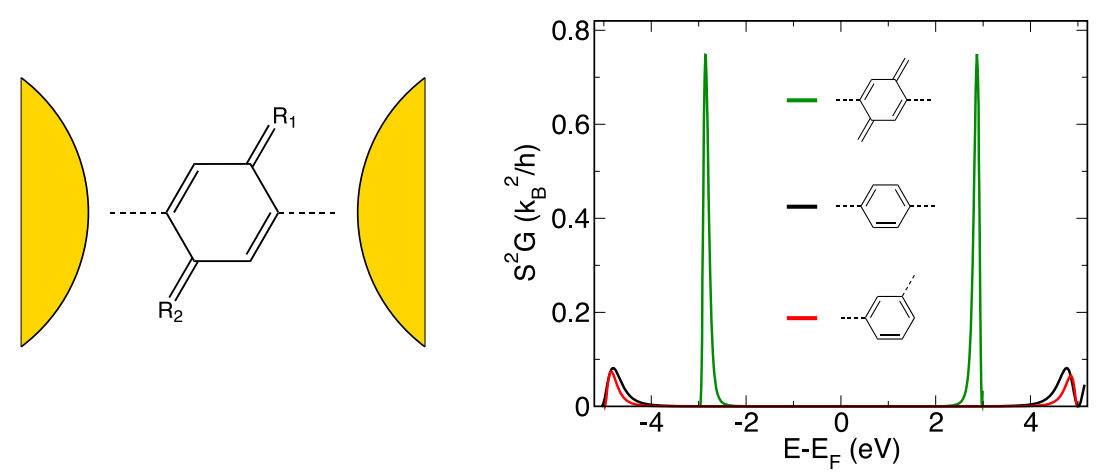

(c)

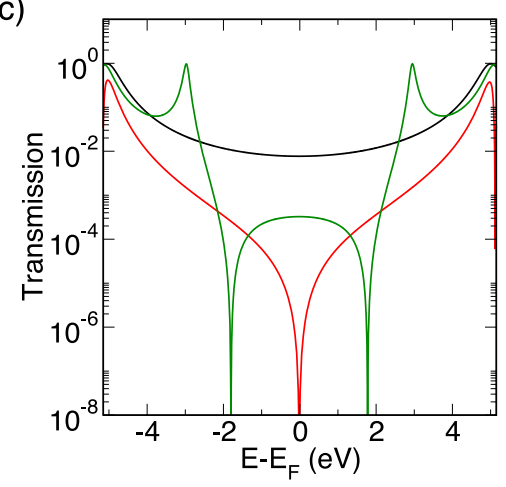

(b)

(d)

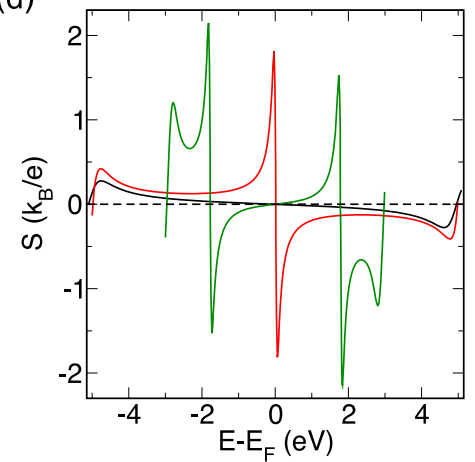

FIG. 1. (a) Sketch of a quinoid molecule sandwiched between metal leads. $R_{1}$ and $R_{2}$ denote the substituent side groups. (b) Powerfactor $S^{2} G$ for a quinoid structure and benzene connected in ortho and meta position. The insets show the chemical structures of the molecules. The results are obtained using a Pariser-Parr-Pople (PPP) model with GW. (c) and (d) show the transmission and thermopower as a function of energy. approximation, ${ }^{25-28}$ gives similar maximal PFs as the DFT calculations. We note that a simple nearest-neighbour tightbinding or Hückel model does not capture the split interference feature. Quinoid type structures typically yield a maximal PF an order of magnitude higher than similar molecules showing DQI in the centre of the HOMO-LUMO gap, such as meta-coupled benzene or simple acyclic cross conjugated molecules.

\section{METHOD}

We use DFT as implemented in the GPAW and ADF codes to provide an quantum chemical description of the charge transport through the molecular junction system. ${ }^{29,30} \mathrm{We}$ also use a more approximate density functional tight-binding (DFTB) method. ${ }^{31}$ The three DFT based methods allow us to get an estimate for how sensitive the results are to the particular implementation details. Molecules were optimised in the gas phase using the LDA exchange correlation (xc) functional in ADF and the $\mathrm{PBE}^{32} \mathrm{xc}$ functional in GPAW. Structures used in the DFTB method were relaxed using the B3LYP xc functional. In all calculations, the molecules were attached to the FCC hollow site of $\mathrm{Au}(111)$ with an Au-S bond length of $2.5 \AA$ (1.83 $\AA$ above the surface). In GPAW, the scattering region supercell was modelled using 3-4 atomic Au layers on both side of the molecule. The number of surface layer atoms varies between $4 \times 4$ and $6 \times 6$ depending on the size the molecule and periodic boundary conditions were used in the transverse directions. The 2D Brillouin zone was samples using $4 \times 4 k$-points. In $\mathrm{ADF}$, the extended molecular region includes $3 \times 3 \mathrm{Au}$ atoms in the surface and no periodic boundary conditions are used in the transverse directions. The Au atoms were frozen in the bulk lattice structure using the DFT derived lattice constant (PBE: $a=4.18 \AA$. LDA: $a=4.08 \AA$ ).

For calculations based on GW, a semi-empirical model Hamiltonian based on the PPP type for describing the $\pi$-system is used. ${ }^{33}$ We use the Ohno parametrisation ${ }^{34}$ with long range two-electron interactions with the parameter $U=10.0 \mathrm{eV}$ describing the Coulomb repulsion and nearest neighbour hopping element of $t=-2.5 \mathrm{eV}$. On site energies are taken relative to carbon, which we set to $\varepsilon_{c}=0$. For the GW calculations, we use a wide band approximation for the leads. More details about the GW method can be found in Refs. 26-28.

Transport properties are for all methods calculated using the Landauer Büttiker transmission formula expressed in terms of Green's functions $\tau(\varepsilon)=\operatorname{Tr}\left[G^{r}(\varepsilon) \Gamma^{L}(\varepsilon) G^{a}(\varepsilon) \Gamma^{R}(\varepsilon)\right]$, where $\Gamma^{\alpha}=i\left(\Sigma_{\alpha}^{r}-\Sigma_{\alpha}^{a}\right)$ is given in terms of the lead $\alpha$ selfenergy $\Sigma_{\alpha}$. We calculate the isothermal conductance in the zero bias voltage limit as $G=G_{0} \int\left(-n_{F}^{\prime}(\varepsilon, T)\right) \tau(\varepsilon) d \varepsilon$, where $n_{F}^{\prime}(\varepsilon, T)$ is the derivative of the Fermi function with respect to energy. $T$ is the temperature and $G_{0}=2 e^{2} / h$ is the unit of quantum conductance, where $h$ and $e$ are Planck's constant and the electronic charge, respectively. We calculate the thermopower in the limit of low temperature drop across the molecule from the transmission as $S=(2 e / h T) \int \varepsilon\left(-n_{F}^{\prime}(\varepsilon, T)\right) \tau(\varepsilon) d \varepsilon / G$. The $\mathrm{PF}$ is calculated as $S^{2} G$ and we use room temperature $T$ $=300 \mathrm{~K}$, see Appendix B for more details. We note that we do not use the Sommerfeld expansion expression for the calculations of the thermopower, since the transmissions functions we consider may have structure on the scale of $\mathrm{k}_{\mathrm{B}} \mathrm{T} .{ }^{35}$ 


\section{RESULTS}

We begin by considering a simple model for a quinoid structure, which captures the essential physical mechanism responsible for interesting thermoelectric properties of quinoids. The schematic structure of a transport junction with a quinoid molecule sandwiched between two leads is shown in Figure 1(a). The connection of the central unit to the leads are indicated by the dashed lines. $R_{1}$ and $R_{2}$ denote substituent side groups. In Figure 1(b), we show the $\mathrm{PF}\left(S^{2} G\right)$ calculated with GW for a PPP model Hamiltonian where $R_{1}$ and $R_{2}$ are taken as $\mathrm{CH}_{2}$ groups, as indicated by the inset. The model includes a single $p_{z}$ orbital per carbon atom all with equal on site energies. For comparison, we also show the PF obtained for benzene coupled in para and meta position. The maximal PF, within the HOMO-LUMO gap, for the quinoid structure is seen to reach a value that is an order of magnitude higher than both meta and para-coupled benzene. In fact, the PF for the quinoid is comparable to the maximal PF that can be obtained from a Lorentzian transmission line shape, $\sim 0.9 \mathrm{k}_{\mathrm{B}}^{2} / h$, by using a width of about $1.1 \mathrm{k}_{\mathrm{B}} T$, see Figure 11(a) in Appendix B.

The PFs can be analysed in terms of the transmission and thermopower as a function of energy, shown in Figures 1(c) and 1(d), respectively. The transmissions for both the quinoid and the meta-coupled benzene ring are seen to be highly suppressed, as compared with para-coupled benzene, for an extended energy region around the Fermi level. This is a result of DQI, as we will discuss in more detail below. DQI also introduces a strong variation of the transmission with energy which in turn yields a high thermopower. This can be seen by considering the linear response Sommerfeld expansion expression for the thermopower $S(\varepsilon) \propto \partial_{\varepsilon} \log [T(\varepsilon)] \cdot{ }^{35}$ The metacoupled benzene ring has a single dip in the transmission at the centre of the HOMO-LUMO gap, while the quinoid structure shows two transmission dips-one dip is close to the HOMO energy while the other dip is close to the LUMO energy. While the thermopower for meta-coupled benzene reaches high values close to the Fermi level, the PF ends up being low because the transmission, and thus $G$, is very low here. The quinoid structure on the other hand has the DQI feature near a transmission resonance giving both a high thermopower and transmission within the same energy region. This combination is responsible for the high PF for the quinoid structure. The para-coupled benzene shows a relatively low thermopower, but relatively high transmission and thus ends up having a PF very similar to the meta-coupled benzene. Finally, we note that the quinoid structure has a smaller HOMO-LUMO gap, which is related to the longer conjugation length as compared with benzene, i.e., it is a confinement effect. The maximal PF value is obtained at an energy located between the HOMO resonance and the dip, which is about $0.2 \mathrm{eV}$ away form the HOMO resonance for all three molecules.

We now analyse how the thermoelectric properties can be improved by adding side groups to the quinoid backbone. The aim is to move the DQI feature close to a transmission resonance (HOMO or LUMO) which leads to a peak in the PF, and to shift that peak towards the metal's Fermi energy.

To probe how the two DQI features depend on the choice of the side groups, we show in Figures 2(a) and 2(b) how varying the on-site energies of the side groups in the model Hamiltonian affects the transmission and PF, respectively. The side group site energies are $0.0 \mathrm{eV}$ for both $R_{1}$ and $R_{2}$ in the top panel, $-4.0 \mathrm{eV}$ for both $R_{1}$ and $R_{2}$ in the middle panel, and -4.0 and $4.0 \mathrm{eV}$ for $R_{1}$ and $R_{2}$ in the lower panel. The negative/positive site energies are meant to mimic the effect of EW/ED side groups. We note that ED groups (EDG) and EW groups (EWG) will in general also tend to shift the on-site energies of the ring through inductive and resonance effects by perturbing the electron density within the ring in the $\sigma$ and $\pi$ - system, respectively. The perturbation of the electron density through the $\sigma$-system is caused by polarisation and is not included in the PPP model. The change in electron density will in turn affect the electrostatic potential and can be seen as a way of chemically gating the molecular backbone.

The position of the transmission dips is seen to shift with the side group energies but the two split QI features are quite robust and stay within the HOMO-LUMO gap. It can be seen that a transmission dip can be moved closer to the HOMO resonance by lowering the side group energies, which leads to an increase in the corresponding PF. This can be rationalised from the approximate expression $S \propto \partial_{\varepsilon} \log [T(\epsilon)]$ for the thermopower, that is, the slope of the transmission as function of energy on a logarithmic scale. The slope of the transmission is seen to increase in Figure 2(a) when on-site energies are varied such that the transmission anti-resonance moves closer to the molecular frontier energy levels, i.e., the transmission has to change from a value of 1 at resonance to 0 at the antiresonance over a smaller energy region. The calculated maximal PF within the HOMO-LUMO gap is high in all cases, and reaches a value of $\sim 1.1 \mathrm{k}_{\mathrm{B}}^{2} / h\left(=316 \mathrm{fW} / \mathrm{K}^{2}\right)$. A similar value (a)

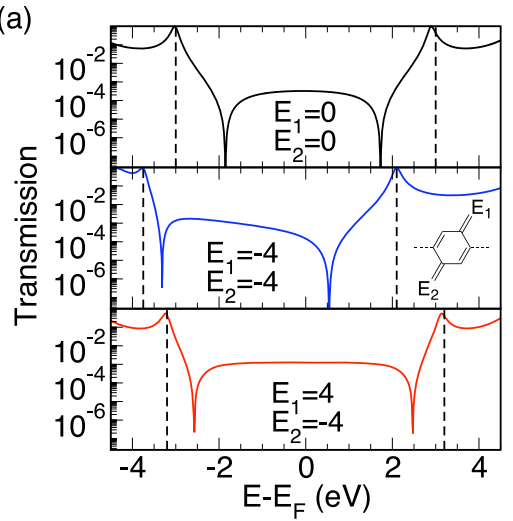

(b)



FIG. 2. (a) Transmissions for the quinoid type structure with on-site energies $\left(E_{1}, E_{2}\right)$ of the $\left(R_{1}, R_{2}\right)$ side groups of $(0.0,0.0)$ top, $(-4.0,-4.0)$ middle, and $(-4.0,+4.0)$ bottom in units of eV. The vertical dashed lines indicate the HOMO and LUMO levels. (b) Same as in (a) but for the power factor, $S^{2} G$. 
was obtained by optimising a general two level model in Ref. 3 . We therefore expect that the EDGs and EWGs can not only be used to tune the position of the interference dips but also to enhance the PF. To experimentally obtain high PFs, molecules are needed where either the HOMO or the LUMO level is close to the Fermi level. This can be chemically controlled by the binding (anchoring) groups, ${ }^{36-38}$ or by mechanical tuning, ${ }^{39}$ as well as by the EW/ED side groups. Before exploring a more detailed quantum chemical description of the molecules shown in Figure 5, we analyse the two split interference features in more detail for the model of the quinoid core structure.

The appearance of the QI features can be analysed in a number of ways ranging from non-spanning nodes to interfering pathways through Feynman path integrals and the phases of molecular orbitals. ${ }^{7,40-43}$ Here, we rationalise the QI interference features in terms of molecular orbitals and corresponding molecular energies. This has the advantage that in the weak molecule lead coupling limit this analysis can be generalised for the correlated electron case by considering so-called Dyson orbitals and energies. ${ }^{44}$

We argue that the central quinoid core structure is responsible for the two transmission nodes. This can be seen either based on graphical rules developed to predict QI in Hückel models ${ }^{11}$ or by systematically reducing the Kohn-Sham Hamiltonian atom by atom as will be discussed below. We note that while a nearest neighbour Hückel model predicts degenerate transmission anti-resonances for equal site energies, ${ }^{12}$ both the GW and the DFT calculations give two well separated anti-resonances for quinoid structures. A similar effect was observed and formulated in terms of long range hopping elements for acyclic cross-conjugated molecules in Ref. 8.

For simplicity, we proceed with an effective non-interacting model where we use the Hückel molecular orbitals but quasi-particle energies taken from the $\mathrm{GW}$ calculations. The use of quasi-particle energies instead of Hückel molecular yields split interference features. ${ }^{44}$ The two central DQI features of the quinoid structure, see Figures 1(a) and 1(c), can be accounted for by considering the four molecular orbitals closest to the Fermi level (HOMO-1, HOMO, LUMO, LUMO+1). This may be seen by first considering the condition for the transmission going to zero, $T(\varepsilon) \propto\left|G_{l r}(\varepsilon)\right|^{2}=0$. Here, the Green's function $G_{l r}$ describes the probability amplitude for an electron or hole propagating through the molecule to a site $l$ from a site $r$ on the molecule, which are connected to the left and right lead, respectively. Then, by expressing $G_{l r}$ in terms of the molecular (uncoupled) Green's function as $G_{l r}(\varepsilon)=G_{l r}^{\mathrm{mol}}(\varepsilon) / D(\varepsilon),{ }^{45}$ we see that a necessary condition for a transmission zero is that $G_{l r}^{\mathrm{mol}}$ is zero for some energy. The denominator in the expression for $G_{l r}$ is given by $D$ $=\left(1-\left[\Sigma_{L}\right]_{l l} G_{l l}^{\mathrm{mol}}\right)\left(1-\left[\Sigma_{R}\right]_{r r} G_{r r}^{\mathrm{mol}}\right)$. In the spectral representation, $G_{l r}^{\mathrm{mol}}$ may be expressed in terms of molecular orbitals $\left\{\psi_{n}\right\}$ and energies $\left\{\varepsilon_{n}\right\}$ as

$$
G_{l r}^{\mathrm{mol}}(\varepsilon)=\sum_{n} \frac{\left\langle l \mid \psi_{n}\right\rangle\left\langle\psi_{n} \mid r\right\rangle}{\varepsilon-\varepsilon_{n}+i \eta},
$$

where $\eta$ is a positive infinitesimal. The elements $\left\langle l \mid \psi_{n}\right\rangle$ and $\left\langle r \mid \psi_{n}\right\rangle$ give the amplitude of the $n$th molecular orbital on site $l$ and $r$, respectively.
From Eq. (1), we infer that the relative sign of the HOMOand LUMO amplitudes on the sites $l$ and $r$ determines the parity of the number of transmission zeros within the HOMOLUMO gap. This is illustrated in Figures 3(a) and 3(b), where we for a few representative cases sketch the real part for of $G_{l r}^{\mathrm{mol}}$ within the HOMO-LUMO gap with an even and odd number of zero crossings, respectively. The contribution to $\operatorname{Re}\left[G_{l r}^{\mathrm{mol}}\right]$ from the HOMO and LUMO diverges when approached from within the HOMO-LUMO gap towards either $+\infty$ or $-\infty$, with the sign determined by the residue $\left.\left(=\left\langle l \mid \psi_{n}\right\rangle\left\langle\psi_{n} \mid r\right\rangle\right\rangle\right)$. All other orbitals only contribute with a finite value to $\operatorname{Re}\left[G_{l r}^{\mathrm{mol}}(\varepsilon)\right]$ within the HOMO-LUMO gap. This means that the function $\operatorname{Re}\left[G_{l r}^{\mathrm{mol}}(\varepsilon)\right]$ can in general be drawn as a continuous line connecting the divergence at $\varepsilon_{\mathrm{HOMO}}$ and $\varepsilon_{\mathrm{LUMO}}$, with the detailed shape determined by all HOMOs and LUMOs. If the residues of the HOMO and LUMO have different signs, we are in the situation corresponding to Figure 3(a), where we see that any continuous line is forced to cross zero and even number $(0,2,4, \ldots)$ of times. With four orbitals, as in our model, only 0 or 2 zero crossings are possible.

If the HOMO and LUMO residues have the same sign, we are in the situation shown in Figure 3(b), where any continuous line is forced to cross zero an odd $(1,3,5, \ldots)$ number of times. From this, a quick assessment and classification of interference features can be made in terms of the HOMO and LUMO orbitals only: Constructive interference between HOMO and LUMO results in an even number of transmission zeros while for destructive interference the number of zeros is odd.

To simplify the analysis even further, we now turn to the Coulson-Rushbrooke (CR) pairing theorem ${ }^{46}$ for alternating hydrocarbons, i.e., molecules which can be viewed as bipartite. The pairing theorem relies on a particle-hole symmetry and states that the molecular energies come in pairs as $\varepsilon_{\text {НОMO- } n}$ $=\varepsilon_{\mathrm{LUMO}+n}$ for $n=0,1,2, \ldots$. The molecular orbitals of such a pair are identical except for a sign change on one of the sublattices, i.e., every other atom, which means that the sign of the residues of the orbitals in a CR pair can be predicted without any calculations. In short, if leads are connected to the same sub-lattice of a molecule the orbitals in a CR pair, they interfere destructively, giving an odd number transmission zeros. For leads connected at sites belonging to different sub-lattices, the two orbitals in a CR pair interfere constructively, giving an even number of transmission zeros. In Figure 4(b), we sketch the four important quinoid frontier molecular orbitals, with the weight on a site represented by the size of the circle and the (a)

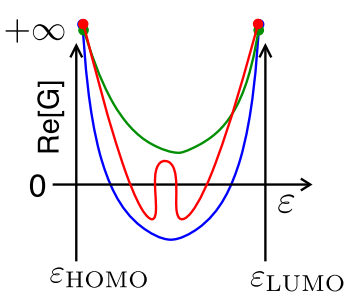

(b)

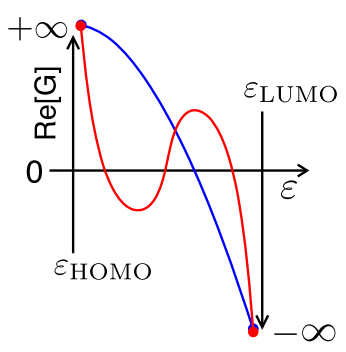

FIG. 3. (a) Sketch of a few representative cases of $\operatorname{Re}\left[G_{l r}^{\mathrm{mol}}\right]$ from HOMO and LUMO orbitals having different relative signs on sites connected to leads. (b) Same as (a) but for HOMO and LUMO orbitals with same sign on sites connected to leads. 
(a)

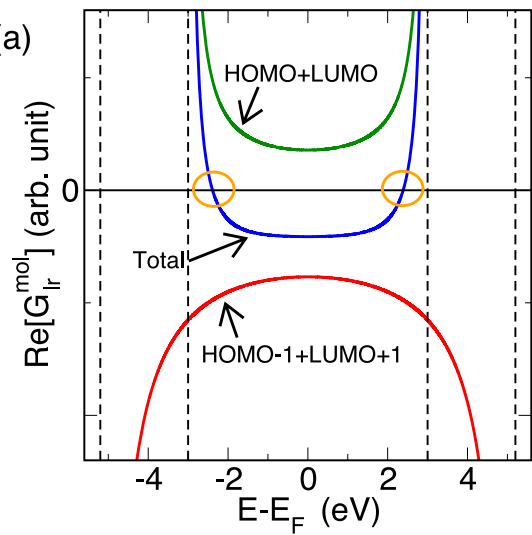

(c)

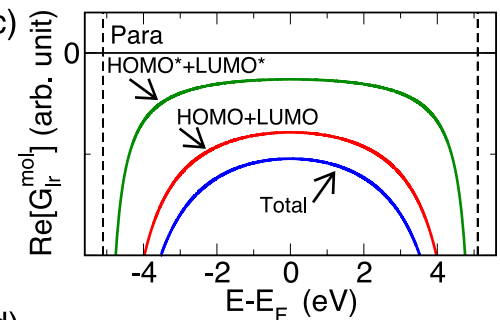

(d)

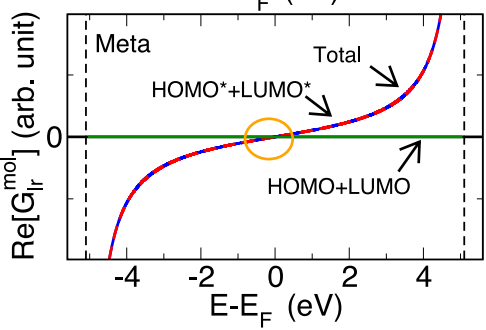

(b)

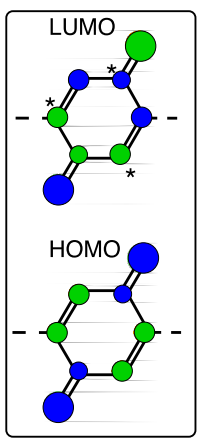

(e)

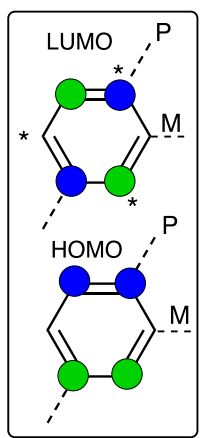

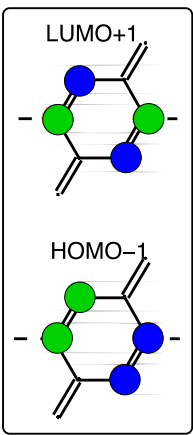

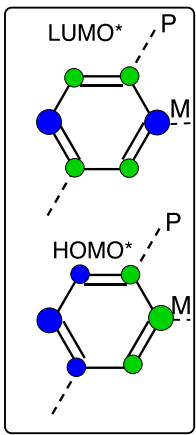

FIG. 4. (a) Contributions to the real part of the molecular Green's function $G_{l r}^{\mathrm{mol}}$ for the quinoid structure; HOMO and LUMO (blue curve) pair and the HOMO-1 and LUMO+1 pair (red curve). The vertical dashed lines indicate the positions of the poles, arising from the molecular energy levels. (b) The molecular orbitals are represented with the weight on the different sites by the size of the circles and the sign by the colour. (c) and (d) Same as (a) but para and meta connected benzene, respectively. (e) Same as (b) but for benzene para. sign by the color. The dashed lines indicate the sites $l$ and $r$ for which leads are connected. The starred sites constitute one sublattice of the molecule. We see that the paired orbitals, namely, the HOMO and LUMO and also the HOMO- 1 and LUMO+1, indeed follow the behaviour predicted by the CR theorem. Due to the close relation between orbitals in a CR pair, we find it advantageous to first sum up the contribution from a CR pair in Eq. (1) and subsequently sum up all pairs.

In order to enable destructive interference in the case where CR-paired orbitals interfere constructively, two or more sets of such paired orbitals are needed, i.e., a minimum of four molecular orbitals. We show the paired HOMO-LUMO (blue line) and the paired HOMO-1 and LUMO+1 (red line) contributions to $\operatorname{Re}\left[G_{l r}^{\mathrm{mol}}\right]$ for the quinoid in Figure 4(a). Here, the vertical dashed lines indicate the positions of the quasiparticle levels. It can be seen that the HOMO-LUMO pair adds up constructively due to the different signs arising from the product of the molecular orbital weights on the $l$ and $r$ sitesa consequence of the leads being connected to different sublattices. In the same way, the HOMO-1-LUMO+1 pair interferes constructively. The destructive interference observed in the total transmission arises when we consider the sum of the pair contributions (green line), which is seen to cross zero twice in accordance with the general classification based on the amplitudes of the HOMO and LUMO orbitals on the sites connected to leads. The transmission nodes are a consequence of the HOMO and LUMO pair having a smaller weight on the $l$ and $r$ sites than the HOMO- 1 and LUMO+1 pair. If we imagine increasing the HOMO and LUMO orbital weight on the lead sites, then at some point, the transmission nodes will disappear, although the transmission will still be suppressed. The DQI is for the quinoid therefore not only a result of the phases of the molecular orbitals. Similarly, the relative position of the HOMO- and HOMO-1 levels (and the LUMO- and LUMO+1 levels) also influences the appearance of transmission zeros.

It is interesting to compare the quinoid to benzene in paraposition, where the orbitals making up a CR pair interfere constructively just as for the quinoid, see Figures 4(c) and 4(e). However, in contrast to the quinoid, adding up the two pairs now leads to constructive interference. This is a result of a different phase relation between the two CR pairs for benzene. For meta-coupled benzene, the CR pairing theorem ensures that the two orbitals making up a CR pair always interfere destructively, resulting in a zero crossing in $\operatorname{Re}\left[G_{l r}^{\mathrm{mol}}(\varepsilon)\right]$ at the Fermi level, see Figure 4(d).

As already mentioned, a nearest neighbour Hückel or tight-binding model predicts a doubly degenerate node and does not capture the characteristic split interference feature of the quinoid structure. ${ }^{12,44}$ In terms of the analysis presented here, which was based on Eq. (1), the doubly degenerate transmission node can be interpreted graphically as in Figure 4(a). $\operatorname{Re}\left[G_{l r}^{\mathrm{mol}}\right]$ (blue line) may be shifted up along the $y$-axis by a change in the molecular energy levels (or a change in orbital weight on $l$ and $r$ ). At some point, the two zero crossings coincide at the Fermi level. This is exactly what happens if 
M1



M2<smiles>Sc1ccc(C#Cc2cc(=C3SC=CS3)c(C#Cc3ccc(S)cc3)cc2=C2SC=CS2)cc1</smiles>

M3<smiles>COC(=O)C1=C(C(=O)OC)SC(=c2cc(C#Cc3ccc(S)cc3)c(=C3SC(C(=O)OC)=C(C(=O)OC)S3)cc2C#Cc2ccc(S)cc2)S1</smiles>

M4<smiles>COC(=O)C1=C(C(=O)OC)SC(=C2C=C(C#Cc3ccc(S)cc3)C(=O)C=C2C#Cc2ccc(S)cc2)S1</smiles>

M5

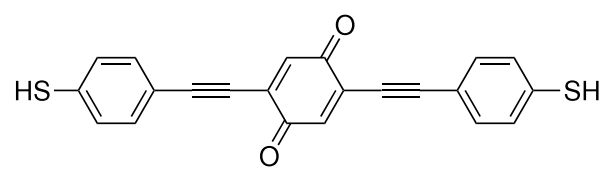

FIG. 5. Molecules M1-M5 with a quinoid type structure consisting of a central cross-conjugated phenyl ring. M1 contains $\mathrm{CH}_{2}$ side groups, M2 contains a TTF electron donating unit, M3 contains a TTF unit with esters, M4 is a possible zwitter-ion with an electron donating DTF unit with ester groups on one side, and an oxygen atom on the other side. Finally, M5 contains two electron withdrawing oxygen atoms.

one uses the tight-binding molecular orbitals and energies to construct $G_{l r}^{\mathrm{mol}}$. The splitting can be obtained by adding a scissors operator to the Hückel Hamiltonian to change the HOMOLUMO gap. The scissors operator will in general have long range hopping matrix elements, which has been shown to split interference features in some cases. ${ }^{8}$

From the four frontier molecular orbitals in Figure 4(b), we can estimate the effect of changing the on-site energies on the side groups. The HOMO- 1 and LUMO+1 have (almost) zero weight on the side group sites and a perturbation of the onsite energies, $\hat{V}_{s g}$, will therefore not change the HOMO-1 and LUMO +1 orbitals and energies to lowest order since $\hat{V}_{s g}|\alpha\rangle$ $\approx 0$ for $\alpha \in\{$ HOMO-1,LUMO+1\}. Thus, the HOMO- and
LUMO levels shift a lot, but the HOMO-1 and LUMO+1 do not. This can be used to give a graphical interpretation in Figure 2(a). A change of $\Delta \varepsilon$ of the side group on-site energies will result in a shift of the HOMO- and LUMO levels of $\Delta \varepsilon$, while the HOMO-1- and LUMO+1 levels stay fixed. This will result in a shift of the position of the two dips in the same direction as the HOMO and LUMO level.

To explore the robustness as well as the tunability of the DQI features with respect to the EW/ED character of the substituent side groups, we have performed quantum chemical calculations based on DFT for the molecules M1-M5 shown in Figure 5.

Before discussing molecules M1-M5 in more detail, we give a comparison between the transmission calculated with the different DFT methods in Figure 6(a) for M1. The two split DQI features discussed for the simple quinoid model above are clearly visible and we emphasise the very good agreement between the GPAW and ADF calculations despite small variations in geometry and the use of different xc functionals. We note that the agreement between the methods, especially the position of the Fermi level, is less good for M2-M5. However, the side group induced changes and trends are not affected and we therefore proceed discussing the results obtained with GPAW.

We stress that we have used flat $\mathrm{Au}(111)$ surfaces for simplicity and not investigated the effect of using different lead structures, such as a small pyramids or add atoms on a flat surface. While the use of different lead structures may certainly change the conductance and the thermoelectric properties, we do not think it will change the trends and conclusions as these are based on a property of the molecule itself.

To make a more direct connection to the minimal $p_{z}$ model for the quinoid core structure, we have performed calculations starting from the molecule M1 and then systematically removed atoms from its arms. The transmissions in Figure 6(b) are obtained by only considering the $p_{z}$ subspace of the KohnSham Hamiltonian of the full calculation for successive truncations of the arms of the molecule M1, while keeping the central cross-conjugated quinoid unit unchanged. The different transmissions correspond to the molecules denoted M1a-M1d shown in Figure 6(c). Wide band leads are attached to the atoms connected with a dashed line. The position of the transmission nodes is seen to be quite insensitive to the arms of the molecule attached to the central quinoid core structure. Only when leads are attached directly to the quinoid core structure do we see a non-negligible change. We stress that in all cases, the qualitative shape of the transmission function remains intact and in good agreement with the GW results in Figure 2(a).

In Figures 7(a) and 7(b), we show the transmission and PF calculated using GPAW (see Appendix A for the corresponding ADF and DFTB transmission results) for the molecules M1M5. The vertical lines indicate the position of the molecular frontier energy levels obtained by diagonalising the molecular subspace (including sulfur).

For all molecules, except M5, we see clear signs of the two split DQI features. Interestingly, M5 only shows a single transmission anti-resonance, which according to the discussion above based on Eq. (1) implies that the HOMO and LUMO orbitals in this case interfere destructively. Taking molecule 

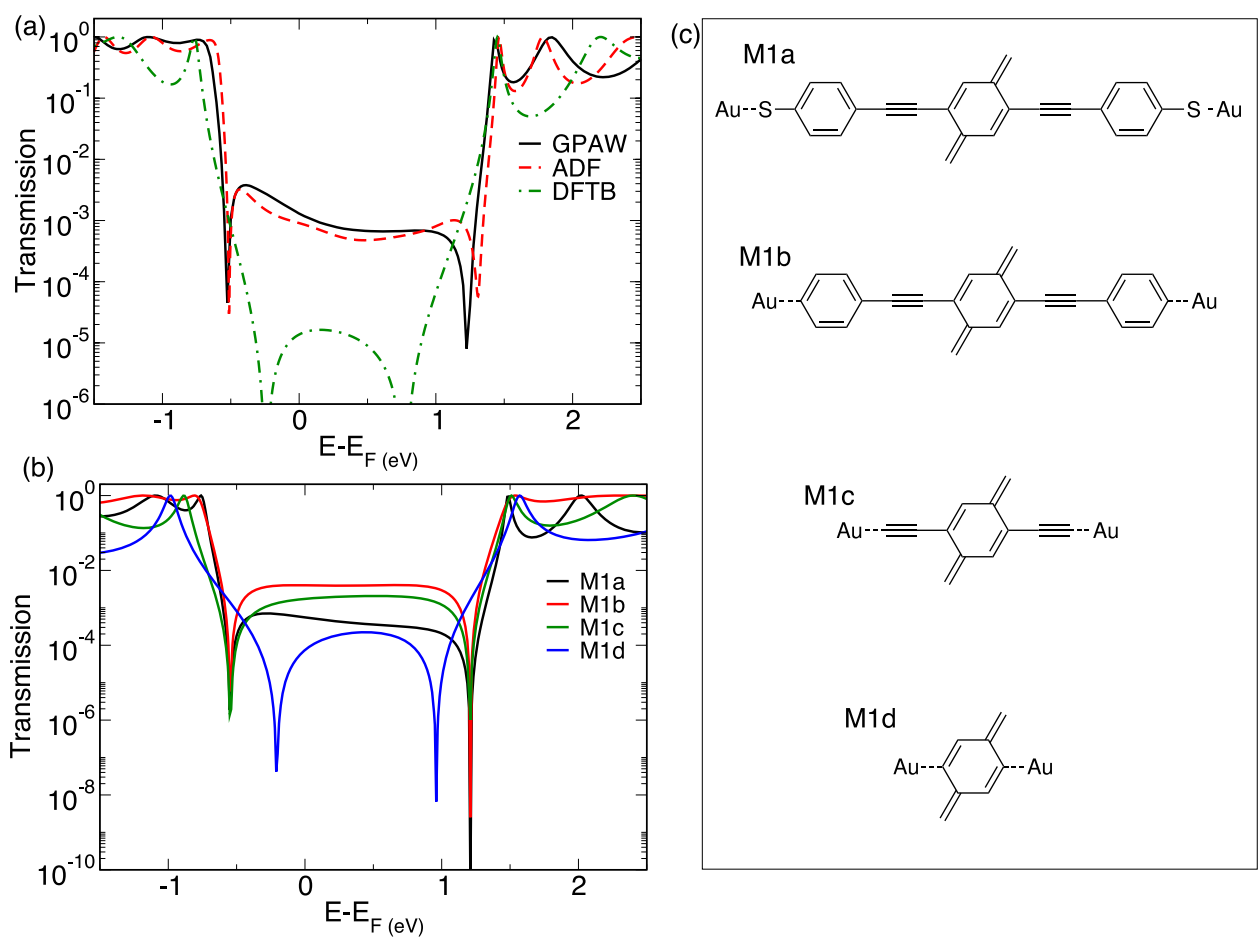

(d)
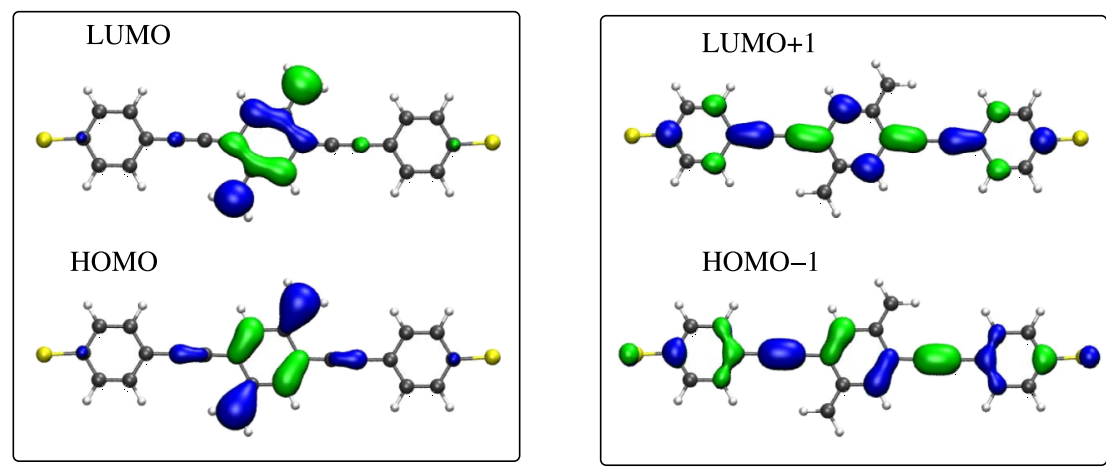

FIG. 6. (a) Transmission as a function of energy for molecule M1 calculated with GPAW, ADF, and DFTB. (b) Transmissions within the $p_{z}$ subspace but for truncated versions of M1 as denoted in (c). Wide band leads are attached at the $p_{z}$ orbitals connected to "Au" with a dashed line in (c). (d) Frontier molecular orbitals for M1.
M1 as the baseline, we observe that the HOMO energy level is closer to the Fermi level than the LUMO energy level. This is typical for molecules bonded to Au via thiols. ${ }^{37}$ The similarity of the shape of the transmission with the results for the simple quinoid model (see Figure 2(a)) is striking. We see two DQI features, one located near the HOMO and the other near the LUMO level. The PF for M1 is shown in the upper most panel in Figure 7(b) and has a maximal value of $\sim 1.5 \mathrm{k}_{\mathrm{B}}^{2} / h$ within the
HOMO-LUMO gap. This is larger than, but comparable to our $\mathrm{GW}$ result for the quinoid model and about $60 \%$ higher than the maximal value of a single level model with a Lorentzian line shape, see Appendix B. Interestingly, the maximal PF for M1 is even larger than the optimized value obtained for a general two-level non-interacting model. ${ }^{3}$ The high PF obtained $0.75 \mathrm{eV}$ below the Fermi level is a result of the transmission anti-resonance being close to a resonance such that both the
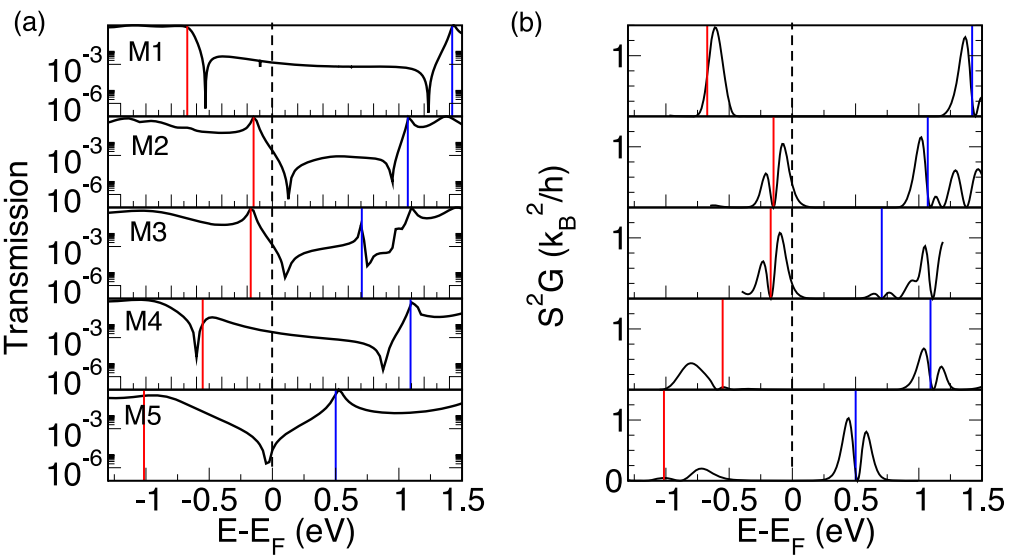

FIG. 7. (a) Transmissions for molecule M1-M5 calculated with DFT (GPAW). (b) Power factor for M1-M5. The vertical colored lines indicate the position of the frontier energy levels of the molecule (HOMO: red, LUMO: blue). 


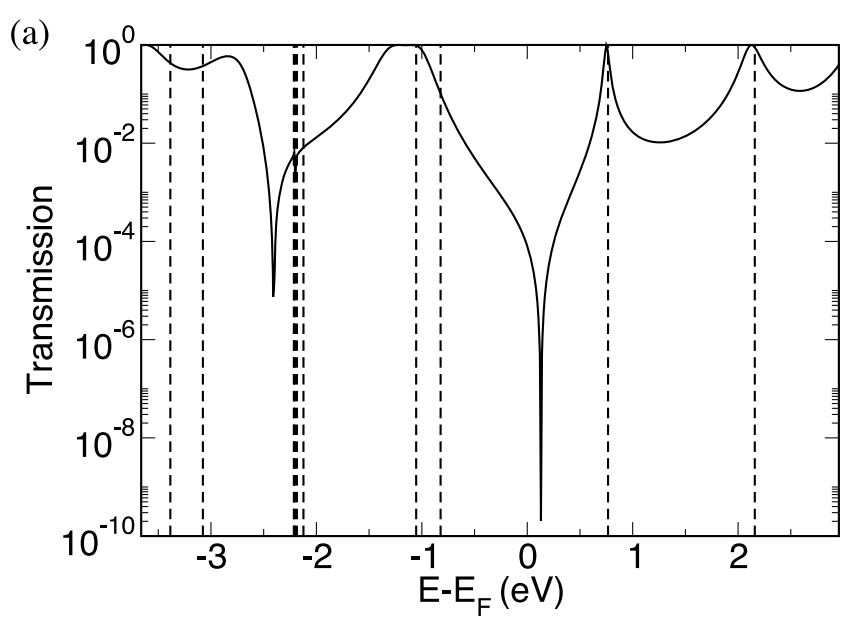

(b)

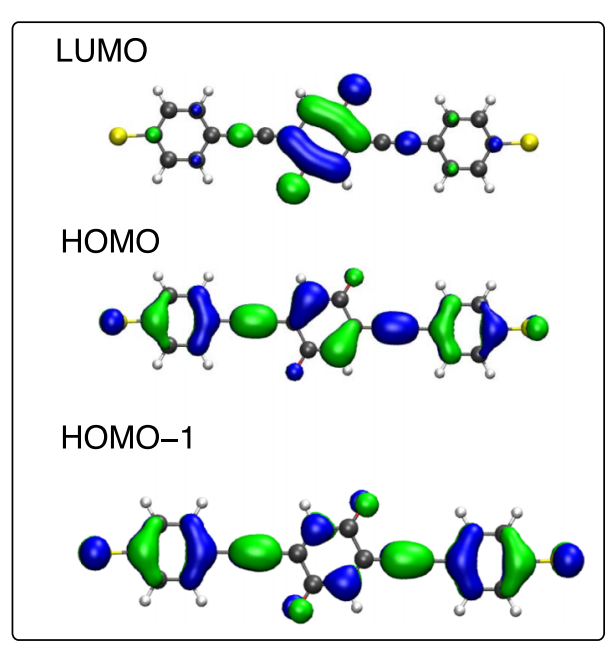

FIG. 8. (a) $\pi$-system contribution to the transmission for M5. The vertical dashed lines indicate the positions of the molecular energy levels. (b) Frontier molecular orbitals for M5.

thermopower and conductance are high at the same energy, as already discussed for the simple quinoid model. In Figure 6(d), we show the frontier molecular orbitals for M1 which are responsible for the two split transmission nodes. The orbitals can be compared with the simple model in Figure 4(b) and are seen to have the same essential features: The HOMO and LUMO orbitals have smaller weight at the connecting sites than the HOMO- 1 and LUMO+1 orbitals, and the orbitals in a CR pair interfere constructively. Also the symmetry of the orbitals with respect to the transport direction is the same. The essential mechanism behind the two DQI features in the DFT calculations is therefore captured by the simple PPP semi- empirical $\pi$-system model for the quinoid core structure. The $\mathrm{PF}$ for M1 at the Fermi level is rather low, suggesting that the band alignment of the molecular levels with the Fermi level of gold is not ideal in terms of possible thermoelectric technological applications.

Molecule M2 contains two electron-rich five membered rings containing sulfur (DTFs) that have an ED character. These units are therefore expected to push the molecular levels up in energy as compared with M1 without the ED rings. Indeed, we see that the HOMO- and LUMO levels are moved up in energy with the HOMO level ending up very close to the Fermi level. We note that with the GPAW code, the HOMO level ends up just below the Fermi level, while it ends up above the Fermi level with the ADF code. We have verified that this is not a basis set issue, by comparing to real space grid calculations performed with GPAW. Based on this, we speculate that this difference is due to use of a cluster model instead of a periodic structure for the leads in ADF. The HOMO-LUMO gap of M2 is considerably reduced as compared with M1. This is an effect of the increased conjugation length of the TTF side groups in $\mathrm{M} 2$ compared with the $\mathrm{CH}_{2}$ side groups in $\mathrm{M} 1$. However, the two split DQI features are still intact and within the HOMO-LUMO gap. While the maximal PF is similar to $\mathrm{M} 1$, the PF at the Fermi level is now relatively high, with a value of $0.4 \mathrm{k}_{\mathrm{B}}^{2} / e\left(\sim 100 \mathrm{fW} / \mathrm{K}^{2}\right)$.

Molecule M3 is similar to M2 but with esters added to the DTF substituents which may have a beneficial effect on the solubility of the molecule. The addition of esters alters the transmission spectra slightly near the Fermi level as compared to M2. However, there are now unoccupied transmission resonances at higher energies, such that the high-energy antiresonance now falls outside the HOMO-LUMO gap. These transmission resonances do not reach a value of 1 and originate from two orbitals, LUMO and LUMO+1, asymmetrically coupled to leads and with most of the weight localised on the $\mathrm{DTF}+$ ester side group. The PF at the Fermi level is again rather high and comparable to what we found for the M2 molecule.

The M4 molecule is an interesting molecule and a possible zwitterion. It contains an EDG (DTF) as one side group and an EWG (atomic oxygen) as the second side group. This gives the molecule a large dipole moment. The transmission as a function of energy shows the two DQI transmission features. The HOMO is coupled rather asymmetrically which results in a transmission resonance with a value below 1 . We see that the opposite character of the two side groups tends to cancel the overall shift of the levels, in agreement with the PPP model studied above, see Figure 2(a).
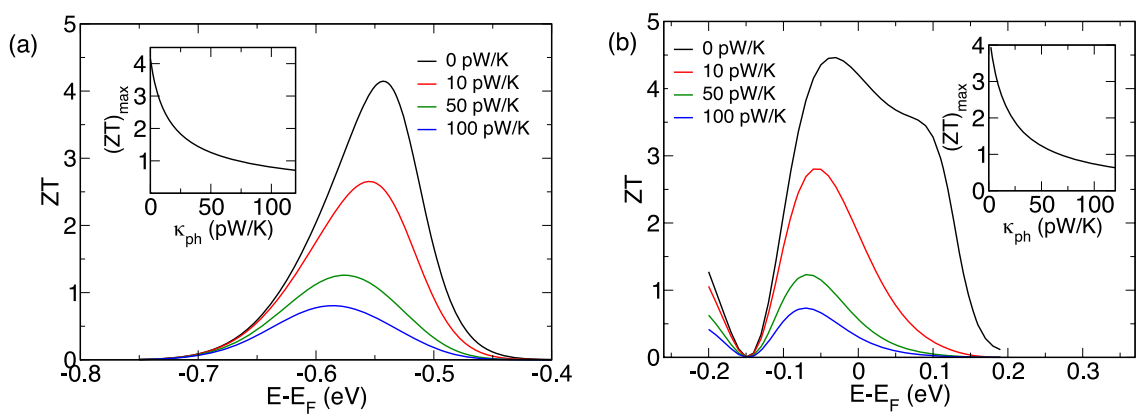

FIG. 9. Figure of merit for M1 and M2 is shown in (a) and (b), respectively. The different lines are obtained by using different values for the phonon thermal conductance $\kappa_{p h}$, as indicated in the legends. The inset shows the maximal value of $\mathrm{ZT}$ within the HOMO-LUMO gap as function of the phonon thermal conductance. 
Molecule M5 has a real quinone core. A similar molecule, namely, the anthraquinone was recently shown to exhibit DQI features in the $\mathrm{dI} / \mathrm{dV} .^{9,10}$ The two electronegative oxygen atoms are EW which is reflected by the molecular levels being drawn down in energy. The LUMO is now closer to $E_{F}$ than the HOMO. There is clearly only one transmission dip within the HOMO-LUMO gap. In Figure 8(a), we show the $\pi$ system contribution to the transmission for M5, from which it is clear that a second interference feature is present at $-2.5 \mathrm{eV}$ below the Fermi level. However, since this is not within the HOMOLUMO gap it is not relevant. By inspecting the frontier molecular orbitals in Figure 8(b) we see that while the LUMO is the same as for M1, the HOMO is not. The symmetry of the HOMO and LUMO orbitals now implies destructive interference within the HOMO-LUMO gap. From the analysis based on Eq. (1), we expect an odd number of transmission zeros within the HOMO-LUMO gap in this case. This is consistent with the observation of one transmission node. The transmission node is positioned close to the Fermi level which induces a strong variation of the transmission function and thus a large thermopower. However, the transmission is suppressed, and thus the resulting PF at the Fermi level is rather low.

To investigate the general thermoelectric performance of the molecules, we show in Figures 9(a) and 9(b) the dimensionless figure of merit ZT as a function of energy for molecule M1 and M2, respectively. To probe the dependence on the phonon thermal conductance, $\kappa_{p h}$, we show the results for four different values ranging from 0 to $100 \mathrm{pW} / \mathrm{K}$. Thermal conductance values in the range $10-100 \mathrm{pW} / \mathrm{K}$ have recently been calculated for similar OPE3 molecules. ${ }^{22}$ Measurements on $n$ Alkanes suggest a similar range of values. We see that maximal ZT, denoted $(\mathrm{ZT})_{\max }$, is almost identical for the two molecules. This is especially clear when comparing the insets which show $(\mathrm{ZT})_{\max }$ as a function of $\kappa_{p h}$. For comparison, the maximal ZT which can be obtained using a thermal conductance of $\kappa_{p h}$ $=10 \mathrm{pW} / \mathrm{K}$ for a single level model with a Lorentzian line shape is about 2.4, see Appendix B. However, in this case, a rather narrow resonance is needed with a with of about $0.3 \mathrm{k}_{\mathrm{B}} T$. The dependence of ZT on energy suggests that molecular levels need to be aligned with the Fermi level with a precision of about $\sim 0.1 \mathrm{eV}$. For molecule M2, the alignment predicted by DFT (GPAW) is relatively good giving high values for ZT at the Fermi level. We have collected $(\mathrm{ZT})_{\max }$ and ZT evaluated at the Fermi level, $(\mathrm{ZT})_{\mathrm{E}_{\mathrm{F}}}$, and $(P F)_{\max }$ in Table I. Here, we clearly see that all the quinoids show promising thermoelectric properties when the maximal values are considered. In terms of the level

TABLE I. ZT and PF for the molecules M1-M5 obtained using DFT-PBE. $\mathrm{ZT}$ is for $\kappa_{p h}=0,10 \mathrm{pW} / \mathrm{K}$. PF is in units of $\mathrm{k}_{\mathrm{B}}{ }^{2} / h\left(\sim 288 \mathrm{fW} / \mathrm{K}^{2}\right)$.

\begin{tabular}{lccc}
\hline Molecule & $(\mathrm{ZT})_{\max }$ & $(\mathrm{ZT})_{\mathrm{E}_{\mathrm{F}}}$ & $(\mathrm{PF})_{\max }$ \\
\hline M1 & $4.1,2.7$ & $0.014,0.001$ & 1.5 \\
M2 & $4.5,2.8$ & $4.2,1.8$ & 1.2 \\
M3 & $4.1,2.7$ & $3.5,1.3$ & 1.1 \\
M4 & $5.5,2.5$ & $0.032,0.004$ & 0.7 \\
M5 & $1.8,1.5$ & $0.706,0.005$ & 1.0 \\
\hline \hline
\end{tabular}

alignment predicted by DFT molecules, M2 and M3 look promising both in terms of power production and efficiency. We note that the level prediction by DFT may not be very accurate, however, we believe that the trend in the level position induced by the substituent side group is a robust feature. Also we have only considered one type of binding structure between the sulfur anchoring group and the gold surface. Both the level positions and the broadening of levels may be sensitive to the details of the gold-sulfur interface and this will in turn affect the calculated thermoelectric properties. However, if it is mainly the broadening of the levels that is affected by the binding geometry, then we do not expect large changes in the calculated properties. This is because the destructive QI forces the transmission to change from 1 to 0 between a frontier orbital resonance and a nearby anti-resonance irrespectively of the broadening.

\section{CONCLUSIONS}

We have shown, based on quantum chemical calculations using DFT as well as GW for an interacting semi-empirical Hamiltonian, that molecules with a quinoid topology may show very high power factors and ZT values, which suggests a high power generation per molecule and good efficiency, respectively.

The good thermoelectric properties were found to originate from a particular DQI feature of quinoid type structures, namely, two split interference features within the HOMOLUMO gap, resulting in a transmission anti-resonance lying close to a molecular resonance. This feature was shown to involve the four nearest frontier molecular orbitals. The split interference feature was found to be rather robust and the position of the resonances and anti-resonances was shown to be highly tuneable by the ED/EW nature of side group substituents. For the five molecules studied, only the real quinone core breaks the two split interference feature, only having a single transmission node in the HOMO-LUMO gap.

DFT calculations showed that the maximal power factor as well as ZT obtainable within the HOMO-LUMO gap was only weakly dependent on the chemical nature of the side group. However, by varying the ED/EW character of the side group, the power factor and ZT values evaluated at the Fermi level can be tuned. Three different DFT based methods predicted the same trends, however, the exact position of the mid HOMOLUMO gap relative to the Fermi level could differ by up to $\sim 0.3 \mathrm{eV}$. The maximal ZT values were predicted by DFT to vary from 1 to 3 , for phonon thermal conductances in the range 10 to $100 \mathrm{pW} / \mathrm{K}$.

The high power factors we predict may be affected by inelastic transport channels, such as those arising from the interaction of electrons with phonons. Here, the current from inelastic processes may be significant, since the HOMO resonance is close to the anti-resonance. An incoming electron with an energy at the anti-resonance may emit a phonon with an energy that brings it close to the HOMO resonance and thereby bypass the anti-resonance. We have neglected such processes, but they appear to be import aspects for further studies of thermoelectric properties of molecules relying on anti-resonances close to resonances. 
(a)

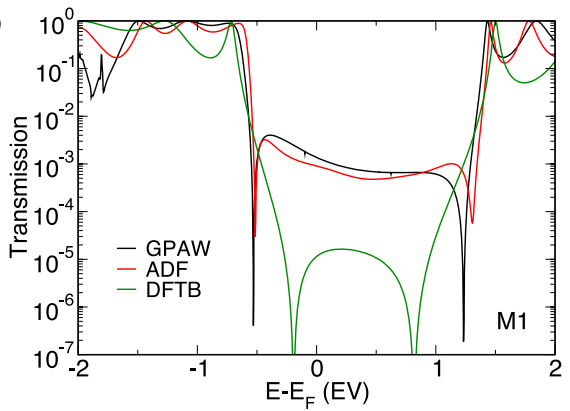

(c)

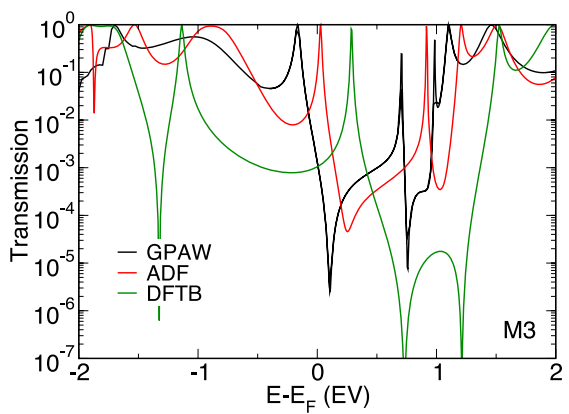

(e)

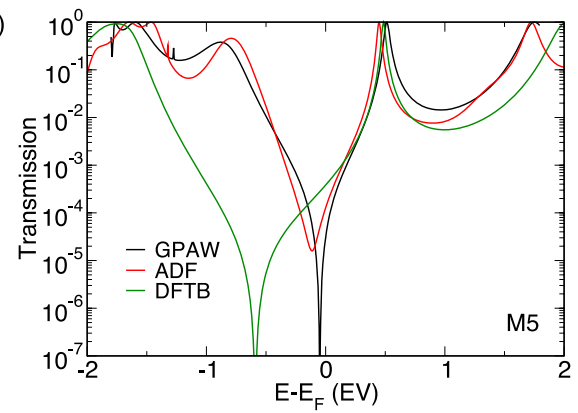

(b)

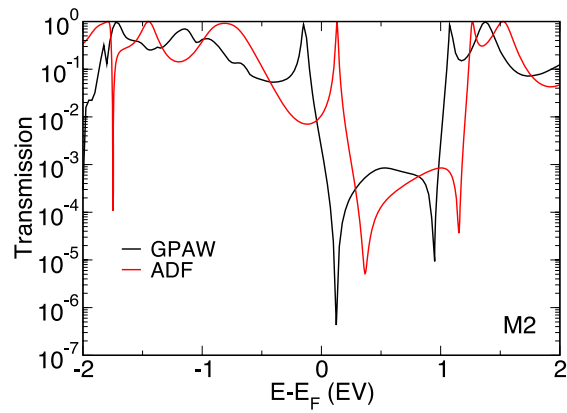

(d)

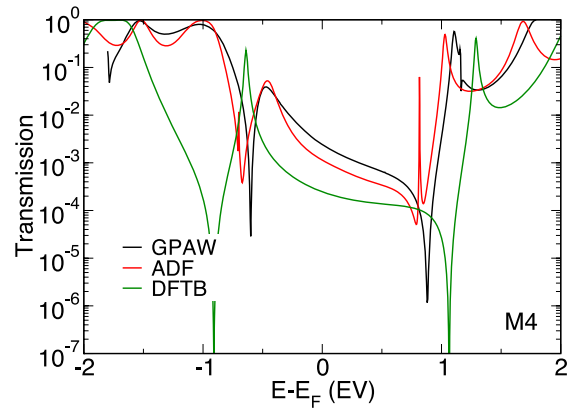

FIG. 10. Transmission calculated using GPAW (black line), ADF (red line), DFTB (green line). (a)-(e) corresponds to molecule M1-M5.

\section{ACKNOWLEDGMENTS}

The authors thank Olov Karlström for valuable discussions on the thermoelectric properties of molecules with destructive interference features. This work was supported by The European Union seventh Framework Programme (FP7/20072013) under the Grant Agreement No. 270369 ("ELFOS"). G.C.S. and M.S. received funding from the European Research Council under the European Union's Seventh Framework Programme (FP7/2007-2013)/ERC Grant Agreement No. 258806.

\section{APPENDIX A: TRANSMISSION COMPARISON}

We compare in Figure 10 the transmission calculated for molecule M1-M5 using GPAW, ADF, and DFTB. We observe that the GPAW and ADF results are in overall good agreement, and that all three methods predict the same qualitative behaviour and trends in accordance with the electron withdrawing and donating character of the substituent side groups.

\section{APPENDIX B: THERMOELECTRICS}

The transport coefficients relevant for thermoelectricity is written as

$$
G=e^{2} L_{0}
$$

$$
\begin{aligned}
S & =\frac{1}{e T} \frac{L_{1}}{L_{0}}, \\
\kappa_{e} & =\kappa_{0}-\frac{1}{T} \frac{L_{1}^{2}}{L_{0}} \\
& =\kappa_{0}-T G S^{2}, \\
\kappa_{0} & =\frac{1}{T} L_{2},
\end{aligned}
$$

in terms of the function

$$
L_{n}(\mu)=\frac{2}{h} \int d \varepsilon(\varepsilon-\mu)^{n}\left(-n_{F}^{\prime}(\varepsilon)\right) \tau(\varepsilon),
$$

where $\left.n_{F}(\varepsilon)=\left(\exp \left((\varepsilon-\mu) / k_{B} T\right)\right)+1\right)^{-1}$ is the Fermi-Dirac distribution function. The thermal electronic conductance $\kappa_{0}$ is for zero chemical potential drop and related to the thermal conductance at zero electric current by $\kappa_{e}=\kappa_{0}-T G S^{2}$.

The dimensionless figure of merit which may be used to characterise the performance of a thermoelectric device is given by

$$
\begin{aligned}
Z T & =\frac{T G S^{2}}{\kappa_{e}+\kappa_{p h}} \\
& =\frac{\kappa_{0}}{\kappa_{p h}}\left(\frac{1-\kappa_{e} / \kappa_{0}}{1+\kappa_{e} / \kappa_{p h}}\right) \\
& \leq \frac{\kappa_{0}}{\kappa_{p h}},
\end{aligned}
$$



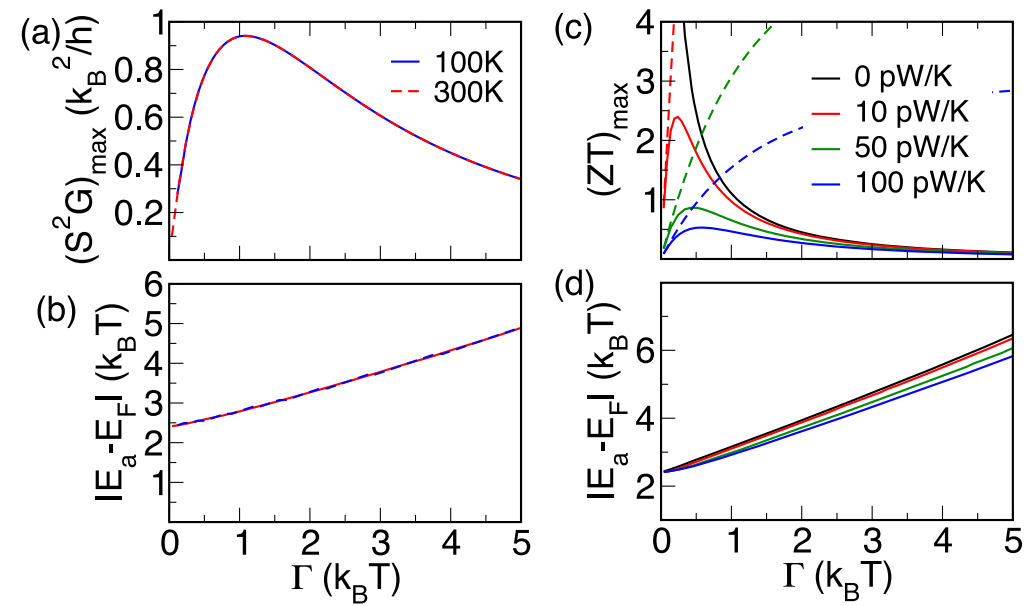

FIG. 11. (a) Maximal power factor as a function of the broadening $\Gamma$ for $T=100 \mathrm{~K}$ (blue line) and $T=300 \mathrm{~K}$ (red dashed line). (b) Level position relative to the Fermi energy giving the maximal power factor in (a) for a given $\Gamma$. (c) Maximal figure of merit as a function of $\Gamma$ for $T=300 \mathrm{~K}$. The labels indicate the values of the contribution to the thermal conductance from phonons. The dashed lines show the MS upper bound. (d) The level position giving the maximal figure of merit in (c) for a given $\Gamma$. which we have rewritten in terms of the thermal conductances in the second line. Since all thermal conductances are larger or equal to zero, we see immediately that the expression in the parentheses is always smaller or equal to 1 . This means that $\kappa_{0} / \kappa_{p h}$ is an upper bound to $Z T$ for a given transmission function and phonon thermal conductance. This upper bound, reached when $\kappa_{e}=0$, is sometimes referred to as the MahanSofo (MS) bound. ${ }^{47} \mathrm{MS}$ showed that the only Dirac delta function as the transmission function gives $Z T=\kappa_{0} / \kappa_{e}$, i.e., the upper bound. However, when concerned with single molecule junctions, then not only is the integral of the transmission bounded but also the value at any energy. The transmission is usually smaller than $\sim 1$ at the relevant energies within the HOMO-LUMO gap. This has the important consequence, that in the case of a infinitesimally narrow transmission resonance $\kappa_{0} \rightarrow 0$. As we shall see below, typically a finite width of the order of $\mathrm{k}_{\mathrm{B}} T$ results in the highest figure of merit, even though this results in a $Z T$ below the MS bound.

Single level model. We now consider a single level coupled to wide band leads for which the transmission takes the simple Lorentzian form

$$
\tau(\varepsilon)=\frac{\Gamma^{2}}{\left(\varepsilon-\varepsilon_{a}\right)^{2}+\Gamma^{2}},
$$

where $\Gamma$ gives the broadening and $\varepsilon_{a}$ is the level position. We assume a temperature of $T=300 \mathrm{~K}$ unless otherwise stated. In Figure 11(a), we show the maximal power factor as a function of $\Gamma$. The level position relative to the Fermi level yielding the maximal power factor for a given $\Gamma$ is shown in Figure 11(b). We have used a temperature of $100 \mathrm{~K}$ (blue line) and $300 \mathrm{~K}$ (dashed red line) which gives the same results. Note that energy is in units of $\mathrm{k}_{\mathrm{B}} T$. The largest value for the maximal power factor is $\sim 0.9 \mathrm{k}_{\mathrm{B}}^{2} / h=258 \mathrm{fW} / \mathrm{K}^{2}$ obtained for $\Gamma \approx 1.1 \mathrm{k}_{\mathrm{B}} T$ and the level positioned about $2.9 \mathrm{k}_{\mathrm{B}} T$ away fro the Fermi level.

We show in Figure 11(c) the maximal value of ZT, denoted as $(\mathrm{ZT})_{\max }$, as a function $\Gamma$ for different phonon thermal conductances, $\kappa_{p h}$, as indicated in the legends. The dashed lines show the MS upper bounds, and we see that only in the limit of small $\Gamma$ does the calculated $(\mathrm{ZT})_{\max }$ approach the MS bounds. However, in this limit, ZT goes to zero. The largest value of $(\mathrm{ZT})_{\max }$ is obtained for a resonance with a width of about $\mathrm{k}_{\mathrm{B}} T / 2$ and the level energy positioned about $2.5 \mathrm{k}_{\mathrm{B}} T$ away from the Fermi level, see Figure 11(d). We observe that while $(\mathrm{ZT})_{\max }$ diverges in the limit of zero broadening for $\kappa_{p h}=0 \mathrm{pW} / \mathrm{K}$ the highest possible value for a finite $\kappa_{p h}$, quickly decreases.

${ }^{1}$ M. Paulsson and S. Datta, Phys. Rev. B 67, 241403 (2003).

${ }^{2}$ J. P. Bergfield, M. A. Solis, and C. A. Stafford, ACS Nano 4, 5314 (2010).

${ }^{3}$ O. Karlström, H. Linke, G. Karlström, and A. Wacker, Phys. Rev. B 84, 113415 (2011).

${ }^{4}$ P. Sautet and C. Joachim, Chem. Phys. Lett. 153, 511 (1988).

${ }^{5}$ C. Patoux, C. Coudret, J.-P. Launay, C. Joachim, and A. Gourdon, Inorg. Chem. 36, 5037 (1997).

${ }^{6}$ M. Mayor, H. B. Weber, J. Reichert, M. Elbing, C. von Hänisch, D. Beckmann, and M. Fischer, Angew. Chem., Int. Ed. 42, 5834 (2003).

${ }^{7}$ G. C. Solomon, D. Q. Andrews, R. P. Van Duyne, and M. A. Ratner, J. Am. Chem. Soc. 130, 7788 (2008).

${ }^{8}$ G. C. Solomon, J. P. Bergfield, C. A. Stafford, and M. A. Ratner, Beilstein J. Nanotechnol. 2, 862 (2011).

${ }^{9}$ C. M. Guédon, H. Valkenier, T. Markussen, K. S. Thygesen, J. C. Hummelen, and S. J. van der Molen, Nat. Nanotechnol. 7, 305 (2012).

${ }^{10}$ V. Rabache, J. Chaste, P. Petit, M. L. Della Rocca, P. Martin, J.-C. Lacroix, R. L. McCreery, and P. Lafarge, J. Am. Chem. Soc. 135, 10218 (2013).

${ }^{11}$ T. Markussen, R. Stadler, and K. S. Thygesen, Nano Lett. 10, 4260 (2010).

${ }^{12}$ T. Markussen, R. Stadler, and K. S. Thygesen, Phys. Chem. Chem. Phys. 13, 14311 (2011).

${ }^{13}$ P. Reddy, S.-Y. Jang, R. A. Segalman, and A. Majumdar, Science 315, 1568 (2007).

${ }^{14}$ K. Baheti, J. A. Malen, P. Doak, P. Reddy, S.-Y. Jang, T. D. Tilley, A. Majumdar, and R. A. Segalman, Nano Lett. 8, 715 (2008).

${ }^{15} \mathrm{~J}$. Balachandran, P. Reddy, B. D. Dunietz, and V. Gavini, J. Phys. Chem. Lett. 3, 1962 (2012).

${ }^{16}$ C. Evangeli, K. Gillemot, E. Leary, M. T. González, G. Rubio-Bollinger, C. J. Lambert, and N. Agraït, Nano Lett. 13, 2141 (2013).

${ }^{17}$ J. R. Widawsky, W. Chen, H. Vázquez, T. Kim, R. Breslow, M. S. Hybertsen, and L. Venkataraman, Nano Lett. 13, 2889 (2013).

${ }^{18}$ Z. Wei, T. Li, K. Jennum, M. Santella, N. Bovet, W. Hu, M. B. Nielsen, T. Bjørnholm, G. C. Solomon, B. W. Laursen et al., Langmuir 28, 4016 (2012).

${ }^{19}$ C. R. Parker, Z. Wei, C. R. Arroyo, K. Jennum, T. Li, M. Santella, N. Bovet, G. Zhao, W. Hu, H. S. J. van der Zant, M. Vanin, G. C. Solomon, B. W. Laursen, K. Nørgaard, and M. B. Nielsen, Adv. Mater. 25, 405 (2013).

${ }^{20}$ J. Fock, M. Leijnse, K. Jennum, A. Zyazin, J. Paaske, P. Hedegård, M. B. Nielsen, and H. S. van der Zant, Phys. Rev. B 86, 235403 (2012).

${ }^{21}$ R. Stadler and T. Markussen, J. Chem. Phys. 135, 154109 (2011).

${ }^{22}$ T. Markussen, J. Chem. Phys. 139, 244101 (2013).

${ }^{23}$ N. Mingo, Phys. Rev. B 74, 125402 (2006).

${ }^{24}$ Z. Wang, J. A. Carter, A. Lagutchev, Y. K. Koh, N.-H. Seong, D. G. Cahill, and D. D. Dlott, Science 317, 787 (2007).

${ }^{25}$ L. Hedin, Phys. Rev. 139, A796 (1965).

${ }^{26}$ K. S. Thygesen and A. Rubio, Phys. Rev. B 77, 115333 (2008).

${ }^{27}$ M. Strange, C. Rostgaard, H. Hakkinen, and K. S. Thygesen, Phys. Rev. B 83, 115108 (2011).

${ }^{28}$ M. Strange and K. Thygesen, Phys. Rev. B 86, 195121 (2012). 
${ }^{29}$ J. Enkovaara, C. Rostgaard, J. J. Mortensen, J. Chen, M. Dulak, L. Ferrighi, J. Gavnholt, C. Glinsvad, V. Haikola, H. A. Hansen, H. H. Kristoffersen, M. Kuisma, A. H. Larsen, L. Lehtovaara, M. Ljungberg, O. Lopez-Acevedo, P. G. Moses, J. Ojanen, T. Olsen, V. Petzold, N. A. Romero, J. StausholmMoller, M. Strange, G. A. Tritsaris, M. Vanin, M. Walter, B. Hammer, H. Hakkinen, G. K. H. Madsen, R. M. Nieminen, J. K. Norskov, M. Puska, T. T. Rantala, J. Schiotz, K. S. Thygesen, and K. W. Jacobsen, J. Phys.: Condens. Matter 22, 253202 (2010).

${ }^{30}$ C. J. O. Verzijl and J. M. Thijssen, J. Phys. Chem. C 116, 24393 (2012).

${ }^{31}$ J. R. Reimers, G. C. Solomon, A. Gagliardi, A. Bilić, N. S. Hush, T. Frauenheim, A. Di Carlo, and A. Pecchia, J. Phys. Chem. A 111, 5692 (2007).

${ }^{32}$ J. P. Perdew, K. Burke, and M. Ernzerhof, Phys. Rev. Lett. 77, 3865 (1996).

${ }^{33}$ J. A. Pople, Trans. Faraday Soc. 49, 1375 (1953).

${ }^{34}$ K. Ohno, Theor. Chim. Acta 2, 219 (1964).

${ }^{35}$ A. M. Lunde and K. Flensberg, J. Phys.: Condens. Matter 17, 3879 (2005).

${ }^{36}$ F. Chen, X. Li, J. Hihath, Z. Huang, and N. Tao, J. Am. Chem. Soc. 128, 15874 (2006).

${ }^{37}$ G. Peng, M. Strange, K. S. Thygesen, and M. Mavrikakis, J. Phys. Chem. C 113, 20967 (2009).
${ }^{38}$ P. Moreno-García, M. Gulcur, D. Z. Manrique, T. Pope, W. Hong, V. Kaliginedi, C. Huang, A. S. Batsanov, M. R. Bryce, C. Lambert, and T. Wandlowski, J. Am. Chem. Soc. 135, 12228 (2013).

${ }^{39}$ M. L. Perrin, C. J. O. Verzijl, C. A. Martin, A. J. Shaikh, R. Eelkema, H. van EschJan, J. M. van Ruitenbeek, J. M. Thijssen, H. S. J. van der Zant, and D. Dulic, Nat. Nanotechnol. 8, 282 (2013).

${ }^{40}$ H.-W. Lee, Phys. Rev. Lett. 82, 2358 (1999).

${ }^{41}$ Y. Tsuji and R. Hoffmann, Angew. Chem., Int. Ed. 53, 4093 (2014).

${ }^{42}$ R. Sawant, J. Samuel, A. Sinha, S. Sinha, and U. Sinha, Phys. Rev. Lett. 113, 120406 (2014)

${ }^{43}$ K. Yoshizawa, T. Tada, and A. Staykov, J. Am. Chem. Soc. 130, 9406 (2008).

${ }^{44}$ K. G. L. Pedersen, M. Strange, M. Leijnse, P. Hedegård, G. C. Solomon, and J. Paaske, Phys. Rev. B 90, 125413 (2014).

${ }^{45}$ C. Caroli, R. Combescot, P. Nozieres, and D. Saint-James, J. Phys. C: Solid State Phys. 4, 916 (1971).

${ }^{46}$ C. A. Coulson and G. S. Rushbrooke, Math. Proc. Cambridge Philos. Soc. 36, 193 (1940).

${ }^{47}$ G. D. Mahan and J. O. Sofo, Proc. Natl. Acad. Sci. 93, 7436 (1996). 